\title{
Une méthode de calcul de la durée de vie à l'amorçage de fissure de fatigue dans un joint soudé en acier S355NL
}

\author{
Nicolas Lautrou ${ }^{a}$, David Thévenet et Jean-Yves Cognard \\ Laboratoire de Mécanique des Structures Navales, ENSIETA, 2 rue F. Verny, 29200 Brest, France
}

Reçu le 15 mars 2007, accepté 7 juin 2007

\begin{abstract}
Résumé - Ce travail porte sur l'étude du comportement en fatigue d'assemblages soudés en acier S355NL, représentatifs d'éléments de structures navales. L'objectif est de développer une méthodologie pour estimer la durée de vie à l'amorçage d'une fissure de fatigue pour ce type de structures. Notre approche se décompose en deux étapes. Premièrement, un calcul de structure par éléments-finis permet d'obtenir les cycles contrainte-déformation stabilisés en tout point de l'assemblage soudé pour un chargement d'amplitude constante ou variable. Ce calcul prend en compte le comportement élastoplastique du métal de base, une variation de la limite d'élasticité dans les différentes zones de la soudure basée sur des mesures de dureté, la géométrie locale du pied de cordon mesurée sur des photos de profil des éprouvettes, et éventuellement la présence de contraintes résiduelles. Deuxièmement, dans le cas où une adaptation élastique rapide se produit, un post-traitement permet de calculer la durée de vie à l'amorçage d'une fissure de fatigue, par l'utilisation d'un modèle d'endommagement à deux échelles basé sur les travaux de Lemaitre et al. Les paramètres matériaux de ce modèle ont été identifiés à partir de courbes d'endurance établies pour le métal de base. Pour valider l'approche proposée, des essais de fatigue en flexion quatre points ont été réalisés pour deux rapports de charge $(0,1$ et 0,3$)$, sur des éprouvettes soudées réalisées par DCN. Pour compléter l'analyse, des mesures de contraintes résiduelles présentes dans les éprouvettes soudées ont été réalisées par diffraction de rayons X. Les comparaisons entre les durées de vie expérimentales et les durées de vie calculées sont encourageantes pour la campagne d'essais réalisée. L'exploitation de la méthode proposée est à l'étude pour un autre mode opératoire de soudage.
\end{abstract}

Mots clés : Amorçage de fissure de fatigue / joints soudés / adaptation / modèle à 2 échelles

Abstract - A fatigue crack initiation life calculation method for a S355NL steel welded joint. This work deals with the fatigue behavior of S355NL steel welded joints of naval structures. The approach suggested here, in order to estimate the fatigue crack initiation life, can be split into two stages. Firstly, stabilized stress-strain cycles are obtained in any point of the welded joint by a finite element analysis, taking constant or variable amplitude loadings into account. This calculation takes account of: base metal elastic-plastic behavior, variable yield stress based on hardness measurements in the various zones of the weld, local geometry at the weld toe measured from specimen photographs, and residual stresses if any. Secondly, if a fast elastic shakedown occurs, a two-scale damage model based on Lemaitre et al.'s work is used as a postprocessing in order to estimate the fatigue crack intiation life. Material parameters for this model were identified from two S-N curves established for base metal. As a validation, four point bending fatigue tests were carried out on welded specimens provided by DCN. Two load ratios were considered: 0.1 and 0.3. Residual stress measurements by X-ray diffraction completed this analysis. Comparisons between experimental and calculated fatigue lifes are promising for the considered loadings. An exploitation of this method is planned for another welding process.

Key words: Fatigue crack intiation / welded joints / elastic shakedown / two-scale damage model

\section{Introduction}

Les structures navales et offshore sont soumises à des sollicitations cycliques d'amplitude variable

\footnotetext{
a Auteur correspondant : lautroni@ensieta.fr
}

principalement dues à la houle. Ces chargements cycliques peuvent provoquer un endommagement par fatigue dans ces structures, principalement dans les joints soudés qui sont les parties les plus sollicitées et qui présentent des contraintes résiduelles liées au procédé de fabrication. 


\section{Nomenclature}

\begin{tabular}{|ll|}
\hline$M_{\max }$ & valeur maximale du moment de flexion \\
$R$ & rapport de charge \\
$\sigma$ & tenseur des contraintes \\
$f$ & surface seuil de plasticité \\
$J_{2}(\sigma)$ & $2^{\mathrm{e}}$ invariant du tenseur des contraintes \\
$X_{1}, X_{2}, X$ & variables d'écrouissage cinématique \\
$R_{1}, R_{2}$ & variables d'écrouissage isotrope \\
$\sigma_{y}$ & limite d'élasticité \\
$p$ & déformation plastique cumulée \\
$\epsilon^{m}$ & module d'Young \\
$\nu$ & coefficient de Poisson \\
$\epsilon$ & tenseur des déformations \\
$\epsilon^{\mathrm{e}}$ & tenseur des déformations élastiques \\
$\epsilon^{\mathrm{p}}$ & tenseur des déformations plastiques \\
$\epsilon^{m}$ & tenseur des déformations dans la matrice \\
$D$ & variable d'endommagement isotrope \\
$\tilde{\sigma}$ & contrainte effective \\
$\mathbf{1}$ & tenseur identité d'ordre 2 \\
$Y$ & taux de restitution d'énergie \\
$\sigma^{\mathrm{d}}$ & partie déviatorique du tenseur des contraintes \\
$D_{\mathrm{c}}$ & endommagement critique \\
$p_{\mathrm{D}}$ & seuil d'endommagement \\
$\sigma_{\mathrm{f}}$ & limite de fatigue \\
$\sigma_{\mathrm{a}}$ nom & amplitude de contrainte nominale \\
\hline &
\end{tabular}

Ce travail porte sur l'étude du comportement en fatigue d'un assemblage soudé bout à bout représentatif des structures navales. Le métal de base de cet assemblage est un acier couramment utilisé en construction navale, le S355NL. Les éprouvettes ont été découpées dans des plaques de $12 \mathrm{~mm}$ d'épaisseur réalisées par DCN. Les 3 passes du cordon de soudure, sous procédé MAG semiautomatique, sont toutes réalisées du même côté dans un chanfrein de préparation à $50^{\circ}$, sur une latte support en céramique et sans bridage. Un soin particulier a été porté à la qualité des cordons de soudure et des radiographies sous rayons $\mathrm{X}$ ont permis de vérifier l'absence de défauts initiaux de soudage.

La méthode développée pour calculer la durée de vie à l'amorçage d'une fissure de fatigue dans ces joints soudés se décompose en deux étapes. Premièrement, un calcul de structure non-linéaire par éléments-finis permet d'obtenir les cycles contrainte-déformation en tout point de l'assemblage soudé, en prenant en compte : un chargement cyclique d'amplitude constante ou variable, le comportement non-linéaire du matériau, une variation de la limite d'élasticité dans les différentes zones de la soudure et la géométrie locale du cordon de soudure. Deuxièmement, un post-traitement permet, dans le cas où une adaptation élastique rapide se produit, de calculer la durée de vie à l'amorçage d'une fissure de fatigue, en utilisant un modèle d'endommagement à deux échelles initialement proposé par Lemaitre et al. [1].

\section{Caractérisation}

Pour cette étude, différentes caractéristiques mécaniques ont été mesurées : le comportement élastoplastique du métal de base, les variations de dureté, la géométrie du cordon de soudure, les contraintes résiduelles présentes à la surface des éprouvettes. Des essais de fatigue en flexion quatre points ont été réalisés sur des éprouvettes soudées.

\subsection{Comportement élastoplastique}

Le comportement élastoplastique du métal de base (acier S355NL) a été déterminé par des essais de traction monotone (thèse de El Malki [2]) et par des essais de traction compression cyclique réalisés en déformation imposée. Ces essais de traction compression cyclique ont été réalisés sur une machine de traction servohydraulique d'une capacité de $100 \mathrm{kN}$, à l'air, en déformation imposée, à température ambiante. Ces essais ont été réalisés avec des éprouvettes cylindriques, comportant une zone calibrée de $15 \mathrm{~mm}$ de longueur et de $6 \mathrm{~mm}$ de diamètre, pour plusieurs amplitudes de déformations et pour deux rapports de déformation $\left(-1\right.$ et $\left.-\frac{1}{3}\right)$.

\subsection{Géométrie du cordon de soudure}

La géométrie du cordon de soudure (en particulier : angle et rayon de raccordement) a été mesurée sur des 
photos de profils des éprouvettes soudées testées en fatigue. La mesure du profil consiste en trois étapes [3] : recherche du point d'intersection entre la tôle et le cordon de soudure; détermination du rayon du plus grand cercle passant par ce point d'intersection et ne pénétrant pas la matière; mesure de l'angle entre les tangentes aux deux points de contact cercle-profil les plus éloignés du point d'intersection, appelé angle de raccordement. À cause du procédé de soudage, la géométrie est plus sévère (i.e. angles et rayons de raccordement plus petits) du côté de la première passe que du côté de la dernière passe. Par la suite, nous appellerons « dessous» le côté de la première passe et « dessus » le côté de la dernière passe. Une modélisation de cette géométrie sera présentée dans la section 3.3 .

\subsection{Mesures de dureté}

Des mesures de dureté ont été réalisées sur 5 échantillons de soudure prélevés dans les plaques. Plusieurs filiations de mesures ont permis de mesurer l'évolution de la dureté dans les différentes zones de la soudure : métal de base, Zone Affectée Thermiquement (ZAT), et métal fondu. Une représentation de la carte des duretés sera présentée dans la section 3.2.

\subsection{Contraintes résiduelles}

En l'absence d'information sur les contraintes résiduelles, nous avons d'abord choisi de détentionner une partie des éprouvettes soudées, par un maintien à $600{ }^{\circ} \mathrm{C}$ pendant $30 \mathrm{~min}$. Ensuite, des mesures de contraintes résiduelles en surface, par diffraction de rayons $\mathrm{X}$, ont montré que celles-ci ne sont pas très importantes [4].

\subsection{Essais de fatigue}

L'absence de bridage au cours de l'opération de soudage induit un « désalignement » angulaire. Afin d'éviter un « redressement » des éprouvettes, indispensable pour des essais de traction compression, nous avons opté pour des essais de fatigue en flexion quatre points. Ces essais ont été réalisés sur une machine de traction compression servo-hydraulique INSTRON équipée d'un banc de flexion d'une capacité de $100 \mathrm{kN}$, à l'air, à température ambiante et à force imposée. Les éprouvettes avaient une largeur de $50 \mathrm{~mm}$ pour une épaisseur de tôle de 11,25 mm.

La différence de géométrie entre les deux côtés (dessus et dessous) de la soudure, nous a permis de réaliser des essais de fatigue dans deux configurations différentes. Dans la première configuration, dite « endroit », le dessus de la soudure est sollicité en traction ondulée, tandis que le dessous est sollicité en compression ondulée. Dans la deuxième configuration, dite « envers », c'est l'inverse.

En l'absence de moyen de détection d'amorçage de fissure, nous avons utilisé un critère d'arrêt d'essai sur le déplacement. Les essais sont réalisés à force imposée, la diminution de rigidité de l'éprouvette due à l'apparition d'une fissure se traduit par une augmentation du déplacement. L'essai est arrêté lorsque cette augmentation atteint une valeur seuil. Pour les niveaux d'effort employés les déplacements maximum étaient de l'ordre de 0,5 à $1 \mathrm{~mm}$, nous avons choisi une valeur seuil de 0,1 à $0,2 \mathrm{~mm}$.

Les éprouvettes ont ensuite été rompues à basse température afin de révéler le profil de fissuration ce qui a permis de relever les dimensions de la fissure de fatigue à l'arrêt de l'essai.

\subsection{Estimation de la durée de propagation}

Afin de se rapprocher de la durée de vie à l'amorçage de fissure, la durée de propagation (depuis une fissure de longueur initiale forfaitaire jusqu'à la longueur de fissure mesurée après arrêt de l'essai) a été estimée pour chaque éprouvette. Pour cela nous utilisons les outils classiques de la Mécanique Linéaire de la Rupture : loi de Paris [5], calcul du facteur d'intensité de contrainte à l'aide des formules de Raju et Newman [6] en flexion associée à une fissure traversante ou de surface. Les données matériaux sont celles du métal de base pour la configuration endroit et celles du métal fondu pour la configuration envers $[2,7]$.

Le tableau 1 présente les durées de vie expérimentales et les durées de propagation estimées, en configuration endroit et envers respectivement.

En configuration « endroit », les durées de vie sont systématiquement supérieures pour les éprouvettes brutes de soudage, par rapport aux éprouvettes détentionnées. L'écart semble peu significatif, mais pourrait s'expliquer, soit par la présence de contraintes résiduelles faibles mais plutôt en compression en pied de cordon côté dessus [4], soit par une géométrie légèrement différente. En effet, les éprouvettes brutes et les éprouvettes détentionnées ne proviennent pas de la même plaque soudée.

En configuration « envers », il n'y a pas de différence significative entre les durées de vie pour les éprouvettes brutes de soudage et les durées de vie pour les éprouvettes détentionnées. Ceci peut s'expliquer, soit par des niveaux de contraintes résiduelles faibles, soit par une relaxation importante des contraintes résiduelles due à la plasticité en pied de cordon. La géométrie étant plus sévère du côté dessous que du côté dessus, la plasticité y est plus importante.

Les durées de vie expérimentales présentées dans la section 5.3 prennent en compte ces durées de propagation.

\section{Calcul de structure}

L'objectif du calcul non-linéaire de structure est de déterminer à chaque instant du chargement les champs de contraintes et de déformations dans la structure. On considère des chargements cycliques. Une structure soumise à un chargement cyclique d'amplitude constante est susceptible d'avoir une réponse cyclique stabilisée, après une phase transitoire qui peut durer plusieurs cycles. Au 
Tableau 1. Durées de vie à l'arrêt de l'essai $N$ et durées de propagation estimées $N_{\mathrm{p}}$ pour les éprouvettes brutes de soudage et détentionnées en configurations « endroit » et « envers $»$.

\begin{tabular}{|c|c|c|c|c|c|c|}
\hline & $R$ & $M_{\max }$ & $\begin{array}{r}\text { bl } \\
\left(\times 10^{3}\right.\end{array}$ & cles) & $\begin{array}{l}\text { détent } \\
\left(\times 10^{3}\right.\end{array}$ & $\begin{array}{l}\text { nées } \\
\text { cles) }\end{array}$ \\
\hline & & (N.m) & $N$ & $N_{\mathrm{p}}$ & $N$ & $N_{\mathrm{p}}$ \\
\hline & & 312,5 & & & 1400 & 249 \\
\hline & & 375 & 968 & 176 & 521 & 134 \\
\hline & 0,1 & 375 & 658 & 170 & 327 & 157 \\
\hline$=$ & & 425 & 488 & 134 & 391 & 112 \\
\hline & & 300 & & & 1163 & 273 \\
\hline & 0,3 & 425 & & & 562 & 197 \\
\hline & & 500 & 440 & 99 & 260 & 96 \\
\hline & & 250 & 862 & 205 & 510 & 95 \\
\hline & & 312,5 & 247 & 68 & 436 & 170 \\
\hline & 0,1 & 375 & & & 116 & 49 \\
\hline 0 & & 425 & & & 95 & 39 \\
\hline & & 312,5 & 427 & $\overline{98}$ & 524 & 98 \\
\hline & 0,3 & 375 & 545 & 75 & 254 & 75 \\
\hline & & 425 & 163 & 61 & 146 & 60 \\
\hline
\end{tabular}

cours de cette phase transitoire, il y a généralement des déformations plastiques dans la structure. La réponse stabilisée peut être soit purement élastique (i.e. les déformations plastiques n'évoluent plus), il y a alors adaptation, soit élastoplastique (i.e. les déformations plastiques évoluent de façon cyclique), il y a accommodation.

Partant du principe que s'il n'y a pas adaptation la durée de vie en fatigue sera limitée, nous ne traiterons en post-traitement que les cas présentant une adaptation.

\subsection{Modèle élastoplastique}

Pour représenter le comportement élastoplastique de notre matériau, nous avons choisi d'utiliser un modèle inspiré de ceux présentés par Lemaitre et Chaboche [8], utilisant deux écrouissages isotropes $\left(R_{1}\right.$ et $\left.R_{2}\right)$ et deux écrouissages cinématiques $\left(X_{1}\right.$ et $\left.X_{2}\right)$. La fonction seuil de plasticité $f \leq 0$ et les lois d'évolution des variables d'écrouissage sont :

$$
\begin{aligned}
f & =J_{2}\left(\sigma-X_{1}-X_{2}\right)-R_{1}-R_{2}-\sigma_{y} \\
\mathrm{~d} R_{i} & =b_{i}\left(Q_{i}-R_{i}\right) \mathrm{d} p \quad i=1,2 \\
\mathrm{~d} X_{i} & =\frac{2}{3} C_{i} \mathrm{~d} \epsilon^{\mathbf{p}}-\gamma_{i} X_{i} \mathrm{~d} p \quad i=1,2
\end{aligned}
$$

Les paramètres de ce modèle (Tab. 2) ont été identifiés numériquement par minimisation de l'écart entre la réponse du modèle et les résultats expérimentaux de traction monotone (Fig. 1) et de traction compression cyclique pour un rapport de déformation $R_{\epsilon}=-\frac{1}{3}$ et une amplitude de déformation $\Delta \epsilon=2 \%$ (Fig. 2). Ce modèle élastoplastique permet de décrire de manière satisfaisante à la fois le comportement monotone et le comportement cyclique, y compris pour des amplitudes de déformations inférieures à $2 \%$ et pour des rapports de déformation de -1 .
Tableau 2. Paramètres du modèle élastoplastique.

\begin{tabular}{rlrl}
$E$ & $=210 \mathrm{GPa}$ & $\sigma_{y}$ & $=400 \mathrm{MPa}$ \\
$\nu$ & $=0,3$ & & \\
$b_{1}$ & $=310$ & $b_{2}$ & $=19,4$ \\
$Q_{1}$ & $=-221 \mathrm{MPa}$ & $Q_{2}$ & $=71,6 \mathrm{MPa}$ \\
$C_{1}$ & $=68700 \mathrm{MPa}$ & $C_{2}$ & $=4750 \mathrm{MPa}$ \\
$\gamma_{1}$ & $=391$ & $\gamma_{2}$ & $=19,6$ \\
\hline
\end{tabular}

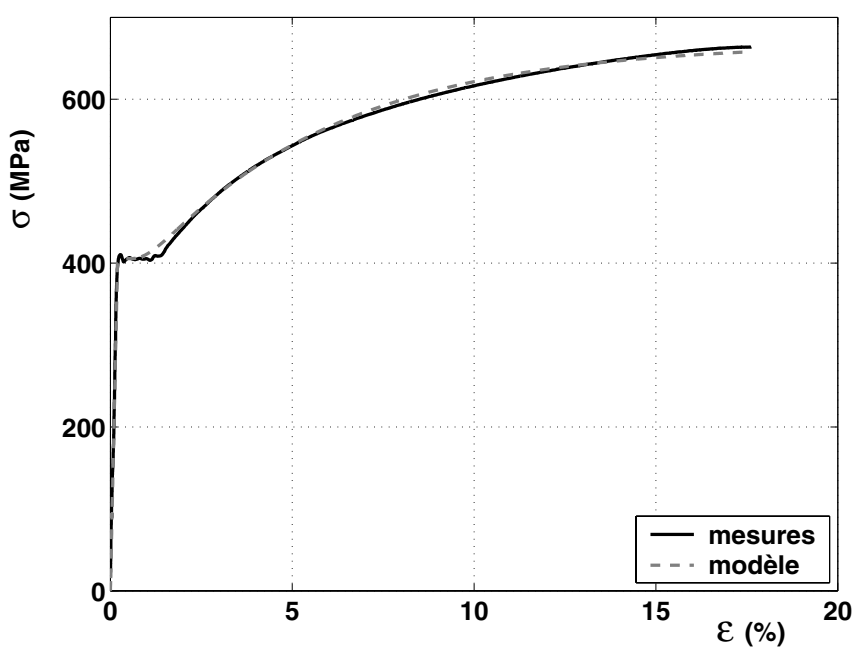

Fig. 1. Réponse du modèle après identification et résultat expérimental en traction monotone.

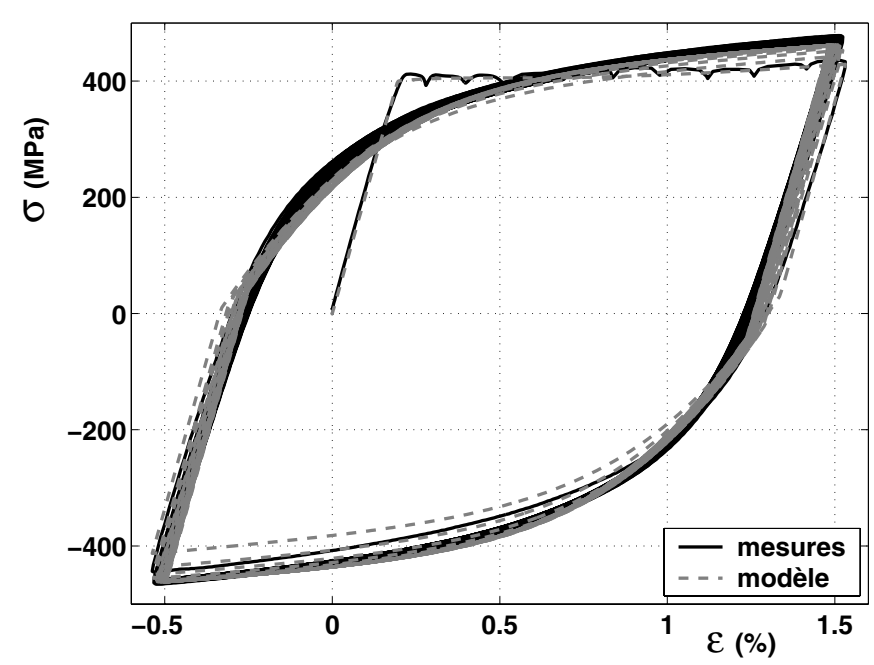

Fig. 2. Réponse du modèle après identification et résultat expérimental en traction-compression cyclique.

\subsection{Modélisation de l'hétérogénéité mécanique}

Nous faisons l'hypothèse que les paramètres d'écrouissage sont identiques dans les différentes zones de la soudure (métal de base, ZAT et métal fondu). Par contre, nous prenons en compte une limite d'élasticité «variable » dans ces différentes zones. Pour cela, nous faisons l'hypothèse de proportionnalité entre la limite d'élasticité et la dureté. Un champ de dureté, basé sur 


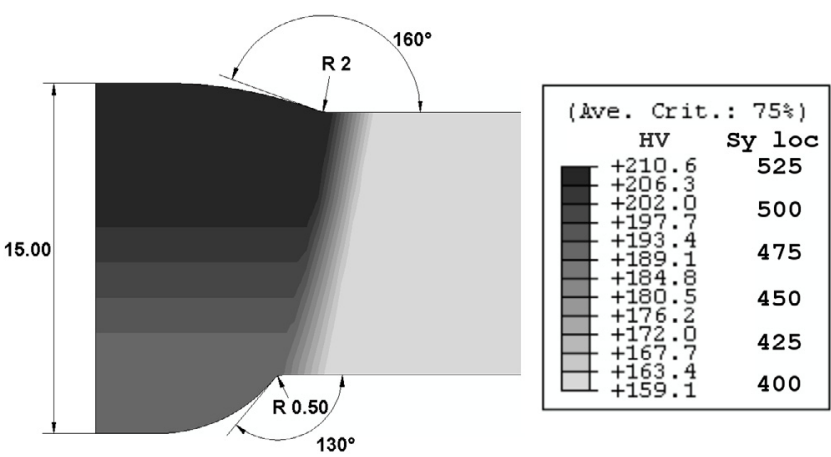

Fig. 3. Champ de dureté introduit dans le calcul et échelle correspondante de limite d'élasticité (Sy loc) pour la géométrie « douce ».

Tableau 3. Valeurs d'angles et de rayons de raccordement et valeurs indicatives de coefficient de concentration de contrainte $K_{\mathrm{T}}$ pour les trois géométries choisies.

\begin{tabular}{|c|rcc|rll|}
\hline géométrie & \multicolumn{3}{|c|}{ dessus } & \multicolumn{3}{c|}{ dessous } \\
& $\begin{array}{rlllll}\text { angle } \\
\left({ }^{\circ}\right)\end{array}$ & $\begin{array}{c}\text { rayon } \\
(\mathrm{mm})\end{array}$ & $K_{\mathrm{T}}$ & $\begin{array}{r}\text { angle } \\
\left({ }^{\circ}\right)\end{array}$ & $\begin{array}{c}\text { rayon } \\
(\mathrm{mm})\end{array}$ & $K_{\mathrm{T}}$ \\
\hline douce & 160 & 2 & 1,5 & 130 & 0,5 & 2,4 \\
moyenne & 150 & 1 & 1,8 & 90 & 0,25 & 3,2 \\
sévère & 140 & 0,3 & 2,5 & 90 & 0,1 & 4,5 \\
\hline
\end{tabular}

les mesures de dureté dans les échantillons soudés, est introduit dans le calcul de structure (Fig. 3).

\subsection{Modélisation de la géométrie}

Les calculs de structures ont été réalisés pour trois géométries (« douce », « moyenne » et « sévère ») se distinguant par les valeurs choisies pour les angles et rayons de raccordement en pied de cordon. Le tableau 3 présente les valeurs d'angles et de rayons pour les trois géométries choisies et à titre indicatif les valeurs de coefficient de concentration de contrainte $K_{\mathrm{T}}$ calculées pour un comportement purement élastique. Pour chacune de ces trois géométries les calculs élastoplastiques ont été menés pour chacun des chargements testés expérimentalement.

\subsection{Modèles de calcul}

Le maillage utilisé pour les éprouvettes soudées en flexion est très fin en surface et aux pieds de cordon (Fig. 4). La première raison est le gradient de contrainte dans l'épaisseur dû au chargement de flexion. La seconde raison est la concentration de contrainte au pied du cordon de soudure due à la géométrie. Pour diminuer le nombre d'éléments, nous utilisons un maillage moins fin dans la zone centrale, dans laquelle les contraintes restent faibles en flexion. La taille des éléments est de l'ordre de $0,5 \mathrm{~mm}$ dans la zone centrale et de l'ordre de $0,03 \mathrm{~mm}$ en surface. Le maillage de l'éprouvette soudée comporte

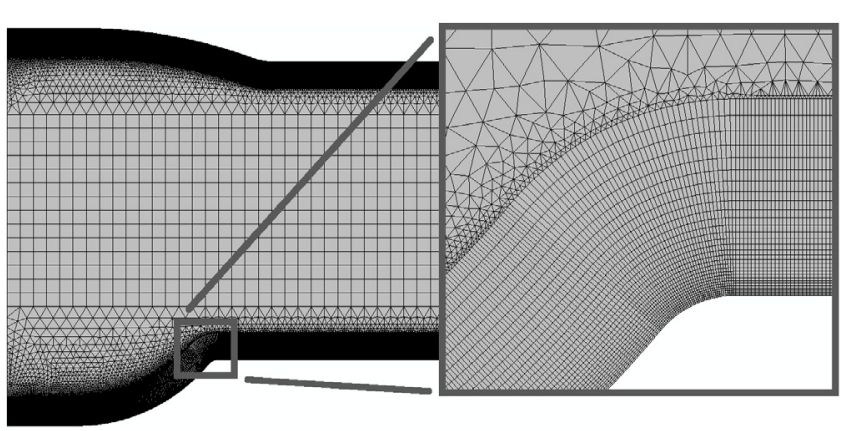

Fig. 4. Maillage pour la géométrie « douce».

environ 350000 éléments (quadrangles et triangles) quadratiques.

Un calcul en 3D avec un tel maillage et une loi de comportement élastoplastique serait beaucoup trop long. Tous les calculs ont été réalisés avec l'hypothèse de contraintes planes.

Les résultats des mesures de contraintes résiduelles, d'une part, les résultats des essais de fatigue, d'autre part, indiquent tous deux que les contraintes résiduelles semblent ici négligeables. Nous avons choisi de ne pas prendre en compte les contraintes résiduelles dans les calculs.

Pour chaque cas de chargement, les 100 premiers cycles ont été calculés.

\subsection{Adaptation de l'éprouvette}

Pour tous les chargements considérés et pour chacune des trois géométries modélisées, des déformations plastiques sont apparues dans les zones les plus sollicitées, i.e. les pieds de cordon. Pour la géométrie « sévère », l'adaptation ne se produit pour aucun des chargements considérés, au cours des 100 cycles calculés. Pour la géométrie « moyenne », l'adaptation se produit pour tous les chargements côté dessus et pour un seul chargement côté dessous. Enfin pour la géométrie « douce », l'adaptation se produit pour tous les chargements côté dessus et pour la moitié des chargements côté dessous.

Le rapport de charge local, une fois l'adaptation atteinte, peut être différent du rapport de charge imposé, à cause de la présence de déformations plastiques qui modifient le niveau de contrainte moyenne. Dans la zone la plus sollicitée, le rapport de charge local varie, suivant les géométries et les chargements, entre $-0,3$ et le rapport de charge imposé $(0,1$ ou 0,3$)$.

Pour les calculs au cours desquels une adaptation se produit, un modèle d'endommagement à deux échelles est utilisé en post-traitement pour estimer la durée de vie à l'amorçage d'une fissure de fatigue. Toutes les composantes du tenseur des contraintes sont utilisées pour le post-traitement qui est effectué pour tous les points du maillage situés à moins de $2 \mathrm{~mm}$ du pied de cordon. 


\section{Amorçage de fissure de fatigue}

En fatigue à grand nombre de cycles, le comportement macroscopique est élastique. La plasticité et l'endommagement se produisent à une échelle inférieure et n'ont pas d'influence sur le comportement macroscopique tant que l'endommagement reste localisé. Le couplage entre déformation et endommagement peut être négligé partout sauf dans le Volume Élémentaire Représentatif (VER) où l'endommagement se produit. Ce constat conduit au modèle à deux échelles proposé par Lemaitre et al. [1,9] que nous utilisons ici en post-traitement.

\subsection{Modèle à deux échelles}

Deux échelles sont introduites : l'échelle mésoscopique (échelle du VER) et l'échelle microscopique (échelle des microdéfauts). Cela conduit à considérer un modèle à deux échelles basé sur la micromécanique d'une inclusion élastoplastique endommageable à l'intérieur d'une matrice élastique.

L'inclusion est plus faible que la matrice par sa limite d'élasticité inférieure à celle de la matrice. La limite d'élasticité de l'inclusion représente la limite de fatigue macroscopique : en dessous de cette limite, il n'y aura ni plasticité ni endommagement dans l'inclusion, il n'y aura pas d'amorçage de fissure de fatigue. Lorsque l'endommagement dans l'inclusion atteint une valeur critique, l'inclusion est rompue, cela correspond à l'amorçage d'une fissure à l'échelle de la matrice.

\subsubsection{Localisation}

Les déformations $\epsilon$ dans l'inclusion sont reliées aux déformations élastiques $\epsilon^{m}$ dans la matrice par la loi de localisation suivante :

$$
\epsilon-\beta \epsilon^{\mathrm{p}}=\epsilon^{\mathrm{e}}+(1-\beta) \epsilon^{\mathrm{p}}=\epsilon^{m}
$$

où $\epsilon^{\mathrm{e}}$ et $\epsilon^{\mathrm{p}}$ sont respectivement les déformations élastiques et plastiques dans l'inclusion, et où $\beta$ est un paramètre issu de l'analyse d'Eshelby [10] d'une inclusion sphérique dans une matrice infinie : $\beta=\frac{2}{15} \frac{(4-5 \nu)}{(1-\nu)}$.

\subsection{2 Élasticité couplée à l'endommagement}

Le couplage entre les déformations et l'endommagement isotrope $D$ est basé sur le concept de la contrainte effective $\tilde{\sigma}=\frac{\sigma}{1-D}$ et le principe d'équivalence en déformation qui conduisent à la loi d'élasticité couplée à l'endommagement :

$$
\epsilon^{\mathrm{e}}=\frac{1+\nu}{E} \tilde{\sigma}-\frac{\nu}{E} \operatorname{Tr}(\tilde{\sigma}) \mathbf{1}
$$

\subsubsection{Limite de fatigue}

La limite d'élasticité de l'inclusion est la limite de fatigue, notée $\sigma_{\mathrm{f}}$. Avec un seul écrouissage (cinématique linéaire) et le critère de Von Mises, la fonction seuil de plasticité s'écrit :

$$
\begin{gathered}
f=J_{2}(\tilde{\sigma}-X)-\sigma_{\mathrm{f}} \\
J_{2}(\sigma)=\sqrt{\frac{3}{2} \sigma^{\mathrm{d}}: \sigma^{\mathrm{d}}} \quad \text { et } \quad \sigma^{\mathrm{d}}=\sigma-\frac{\operatorname{Tr}(\sigma)}{3} \mathbf{1}
\end{gathered}
$$

\subsubsection{Lois d'évolution}

Les lois d'évolution des déformations plastiques $\epsilon^{\mathrm{p}}$, de l'écrouissage cinématique $X$ et de l'endommagement $D$ sont les suivantes :

$$
\begin{aligned}
\mathrm{d} \epsilon^{\mathrm{p}} & =\frac{3}{2} \frac{\tilde{\sigma}^{\mathrm{d}}-X}{J_{2}(\tilde{\sigma}-X)} \mathrm{d} p \\
\mathrm{~d} X & =\frac{2}{3} C(1-D) \mathrm{d} \epsilon^{\mathrm{p}} \\
\mathrm{d} D & =\left(\frac{Y}{S}\right)^{\mathrm{s}} \mathrm{d} p \quad \text { si } \quad p \geq p_{\mathrm{D}}
\end{aligned}
$$

où $p_{\mathrm{D}}$ est le seuil d'endommagement et où $Y$ est le taux de restitution d'énergie défini par :

$$
\begin{aligned}
Y= & \frac{1+\nu}{2 E}\left[\frac{\operatorname{Tr}\left(\langle\sigma\rangle_{+}^{2}\right)}{(1-D)^{2}}+h \frac{\operatorname{Tr}\left(\langle\sigma\rangle_{-}^{2}\right)}{(1-h D)^{2}}\right] \\
& -\frac{\nu}{2 E}\left[\frac{\langle\operatorname{Tr}(\sigma)\rangle^{2}}{(1-D)^{2}}-h \frac{\langle-\operatorname{Tr}(\sigma)\rangle^{2}}{(1-h D)^{2}}\right]
\end{aligned}
$$

où $\langle$.$\rangle est la partie positive d'un scalaire (\langle x\rangle=x$ si $x \geq$ 0 et $\langle x\rangle=0$ si $x<0)$, et où $h$ est un paramètre de fermeture de microdéfauts qui vaut 0,2 pour la plupart des métaux [9].

\subsubsection{Endommagement critique}

Lorsque l'endommagement $D$ atteint une valeur critique $D_{\mathrm{c}}$, il y a amorçage d'une fissure. Cet endommagement critique dépend du chargement et est lié à l'endommagement critique en traction uniaxiale monotone $D_{1 \mathrm{c}}$ par la relation $D_{\mathrm{c}}=D_{1 \mathrm{c}} \frac{\left(\sigma_{\mathrm{u}}\right)^{2}}{2 E Y} \leq 1$, où $\sigma_{\mathrm{u}}$ est la contrainte ultime.

Les paramètres $S$ et $s$ sont respectivement la résistance à l'endommagement et un exposant de nonlinéarité.

\subsubsection{Seuil d'endommagement}

Le seuil d'endommagement $p_{\mathrm{D}}$ dépend du chargement. Le bon indicateur pour prédire l'amorçage de l'endommagement est l'énergie stockée. Pour un chargement cyclique, Desmorat [11] propose de calculer l'énergie stockée 
à la fin de chaque cycle, avec l'hypothèse de plasticité parfaite à la valeur de contrainte maxi $\sigma_{\text {eq }}=$ const. $=$ $\sigma_{\text {eq }}^{\max }$ ainsi qu'à la valeur de contrainte mini $\sigma_{\mathrm{eq}}=$ const. $=\sigma_{\text {eq }}^{\text {min }}$. Pour un chargement cyclique d'amplitude constante, le seuil d'endommagement $p_{\mathrm{D}}$ peut ainsi s'écrire en fonction de la contrainte ultime $\sigma_{\mathrm{u}}$, du seuil d'endommagement en traction monotone $\epsilon_{\mathrm{pD}}$ et d'un exposant de non-linéarité $m$ :

$$
p_{\mathrm{D}}=\epsilon_{\mathrm{pD}}\left[\frac{\sigma_{\mathrm{u}}-\sigma_{\mathrm{f}}}{\frac{1}{2}\left(\sigma_{\mathrm{eq}}^{\max }+\sigma_{\mathrm{eq}}^{\min }\right)-\sigma_{\mathrm{f}}}\right]^{m}
$$

\subsubsection{Sauts de cycles}

Le calcul de durée de vie avec ce modèle se fait par l'intégration pas à pas dans le temps du chargement cyclique jusqu'à amorçage, ou jusqu'à un nombre de cycles maxi ( $10^{9}$ par exemple). Ce calcul étant très long, une procédure de sauts de cycles permet un gain important en temps de calcul.

Pour un chargement cyclique d'amplitude constante, lorsque le comportement est stabilisé, seuls la déformation plastique cumulée $p$ et l'endommagement $D$ évoluent d'un cycle à l'autre. On suppose que $p$ et $D$ évoluent linéairement en fonction du nombre de cycles. Le principe de la procédure de sauts de cycles, inspiré des travaux de Lesne et al. [12], est alors de faire un saut linéaire en tolérant un écart relatif par rapport au résultat d'un saut quadratique.

Une étude de convergence a permis de choisir la valeur du paramètre $\xi$ contrôlant cet écart relatif, pour obtenir un bon compromis entre le temps de calcul et la précision du résultat.

\subsection{Identification des paramètres}

Une partie des paramètres de ce modèle sont identifiés à partir de l'essai de traction monotone : $E=210 \mathrm{GPa}$, $\nu=0,3, C=2000 \mathrm{MPa}, \sigma_{\mathrm{u}}=660 \mathrm{MPa}, D_{1 \mathrm{c}}=0,3$. Les autres paramètres sont identifiés numériquement par minimisation de l'écart entre la réponse du modèle et deux courbes $S-N$ de références établies en traction pour deux rapports de charges différents $(0,1$ et -1$)$ pour le métal de base (Fig. 5) : $\sigma_{\mathrm{f}}=160 \mathrm{MPa}, \epsilon_{\mathrm{pD}}=0,05, m=1,3$, $S=1,5 \mathrm{MPa}$ et $s=2$.

\section{Post-traitement}

En post-traitement des calculs de structure, nous considérons que toutes les zones de la soudure ont le même comportement en fatigue que le métal de base, y compris la limite de fatigue $\sigma_{\mathrm{f}}$.

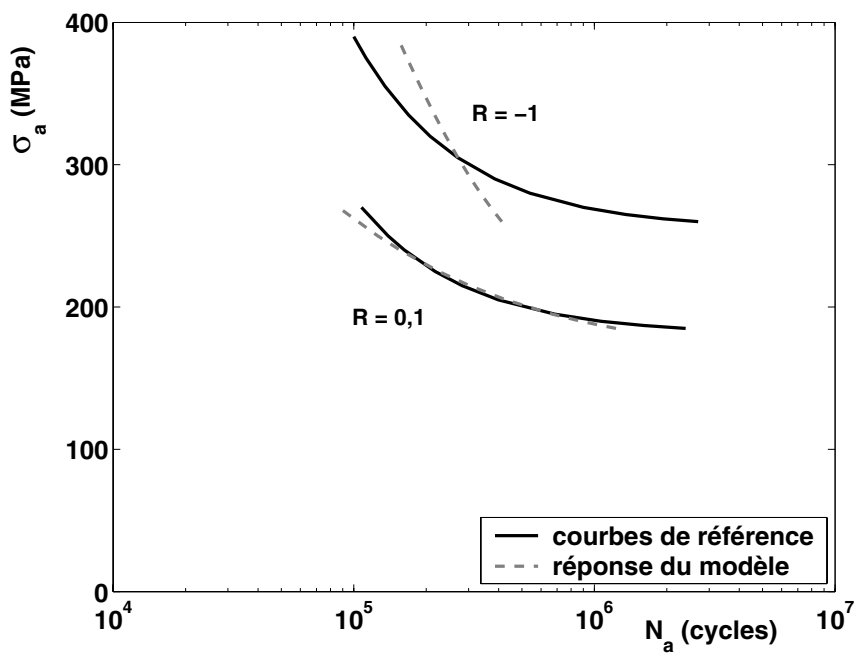

Fig. 5. Courbes $S-N$ de référence et réponse du modèle à 2 échelles après identification des paramètres.

\subsection{Sélection des points}

Le calcul de durée de vie est trop long pour pouvoir être effectué en tout point de la structure. Une première sélection consiste à ne considérer que les points du maillage situés à proximité des pieds de cordon (à moins de $2 \mathrm{~mm}$ ) qui sont les points les plus sollicités. La durée de vie en ces points est alors estimée rapidement en augmentant le paramètre $\xi$ contrôlant les sauts de cycles. Ceci permet d'effectuer de très grands sauts de cycles avec une précision moindre. Une deuxième sélection consiste alors à conserver les 10 ou 20 points dont la durée de vie estimée est la plus courte. Le calcul précis de la durée de vie, avec la valeur du paramètre $\xi$ déterminée précédemment (Sect. 4.1.7), est alors effectué pour ces points.

\subsection{Analyse des zones d'amorçage}

Les points où la durée de vie calculée est la plus courte ne correspondent pas toujours aux sites d'amorçage des fissures ayant provoqué l'arrêt de l'essai de fatigue.

\subsubsection{Côté dessus/côté dessous}

Le modèle à deux échelles prend en compte l'effet de contrainte moyenne. À amplitude de contrainte égale, la durée de vie calculée est plus faible en traction ondulée qu'en compression ondulée.

En configuration « envers », le côté dessous cumule la géométrie la plus sévère et la sollicitation de traction ondulée, le point où la durée de vie calculée est la plus courte se situe systématiquement du côté dessous. En configuration « endroit », il y a compétition entre le côté dessous qui a la géométrie la plus sévère et le côté dessus qui est sollicité en traction, le point où la durée de vie calculée est 
la plus courte se situe parfois du côté dessous et parfois du côté dessus.

Expérimentalement, les fissures qui ont provoqué l'arrêt de l'essai de fatigue se sont systématiquement amorcées du côté de l'éprouvette qui était en traction ondulée. Pour la comparaison entre durées de vie calculées et durées de vie expérimentales (Fig. 6) nous avons retenu systématiquement la durée de vie la plus courte calculée du côté de l'éprouvette qui était en traction ondulée.

Expérimentalement, en configuration « endroit » il est possible que des fissures de fatigue se soient amorcées plus rapidement du côté dessous qui est sollicité en compression mais dont la géométrie est plus sévère que du côté « dessus ». Dans ce cas les fissures amorcées du côté « dessous $»$ se seraient propagées à des vitesses beaucoup plus lentes que les fissures amorcées du côté « dessus », à cause de la sollicitation de compression limitant leur ouverture. Les fissures qui ont provoqué l'arrêt de l'essai sont celles qui, étant du côté sollicité en traction, se sont propagées suffisamment pour diminuer la rigidité de l'éprouvette.

\subsubsection{Sites d'amorçage}

Pour le calcul de durées de vie, nous avons fait l'hypothèse que les paramètres du modèle à deux échelles sont les mêmes dans toute l'éprouvette soudée. Les points où la durée de vie calculée est la plus courte sont par conséquent les points les plus sollicités qui se situent dans le rayon de raccordement en pied de cordon. Ces points se situent dans le métal fondu.

Expérimentalement, les sites d'amorçage des fissures ayant provoqué l'arrêt de l'essai sont à la limite entre le métal fondu et la ZAT.

Aux points où la durée de vie calculée est la plus courte, l'état de contrainte est uniaxial. En effet ces points sont à la surface de l'éprouvette modélisée en deux dimensions avec l'hypothèse de contraintes planes. Cela correspondrait à l'arête d'une éprouvette modélisée en trois dimensions. Les conditions de surface libre imposent un état uniaxial de contrainte en ces points.

\subsection{Durées de vie calculées}

Les figures 6 et 7 montrent une comparaison entre les durées de vie expérimentales et les durées de vie calculées pour les géométries « douce » et «moyenne» dans les deux configurations « endroit $»$ et « envers $»$. Ici, les durées de vie expérimentales sont les durées de vie à l'arrêt de l'essai moins les durées de propagation estimées.

L'effet de la géométrie est très important, pour les durées de vie expérimentales, comme pour les durées de vie calculées. Le choix de la géométrie est donc prépondérant.

Les durées de vie calculées sont généralement inférieures aux durées de vie expérimentales. Seule la durée de vie calculée en configuration endroit pour la géométrie douce et le chargement le plus faible est

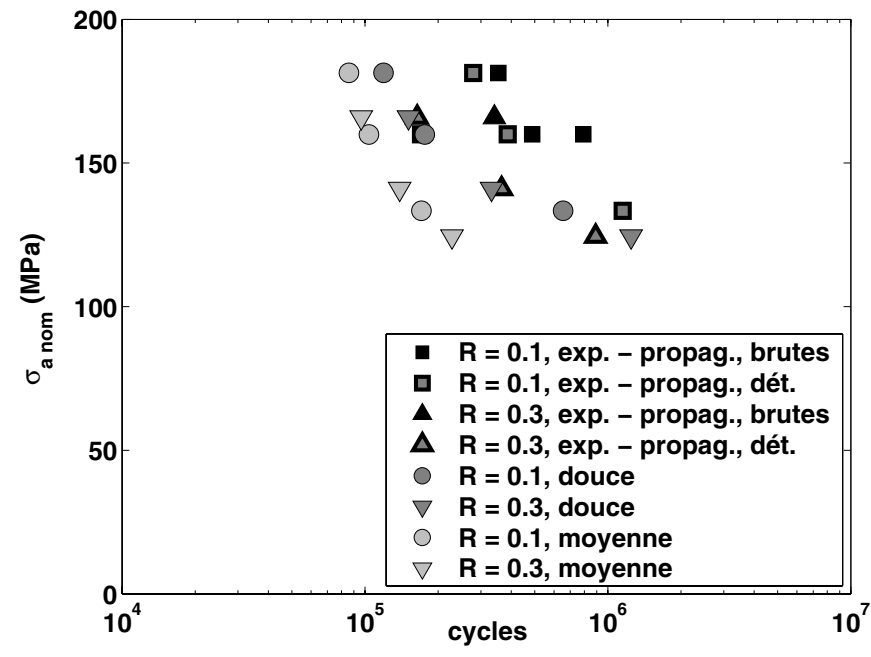

Fig. 6. Comparaison entre durées de vie expérimentales et calculées pour les géométries « douce » et « moyenne », en configuration endroit.

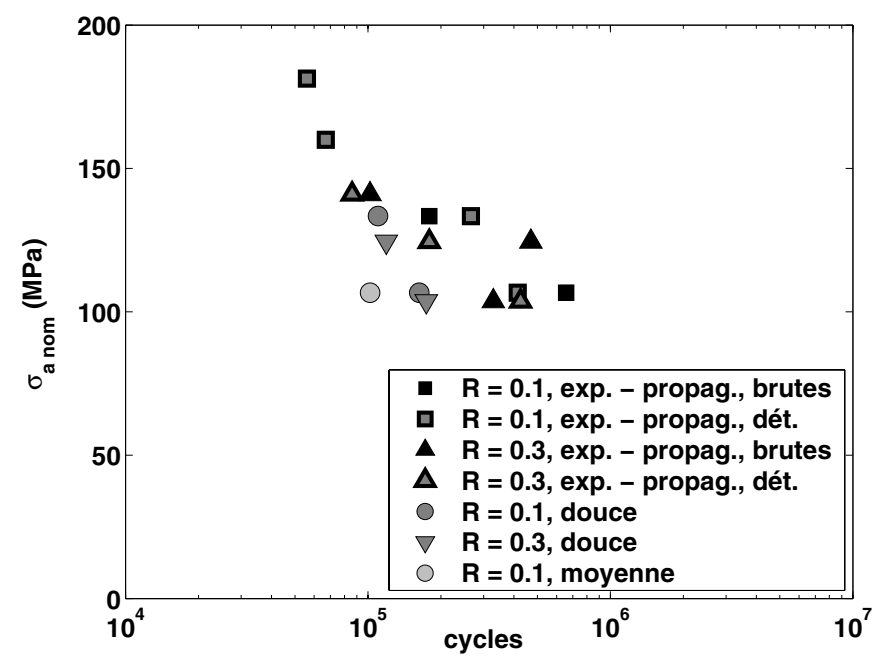

Fig. 7. Comparaison entre durées de vie expérimentales et calculées pour les géométries « douce » et « moyenne », en configuration envers.

supérieure à la durée de vie expérimentale correspondante.

Quelle que soit la configuration, l'écart entre les durées de vie calculées et les durées de expérimentales peut atteindre un facteur 3 pour la géométrie douce et un facteur 7 pour la géométrie moyenne.

\subsection{Discussion}

Pour les modélisations réalisées, plusieurs des hypothèses que nous avons faites sont conservatives.

En supposant que toute la soudure a les mêmes caractéristiques de résistance à la fatigue que le métal de base, les points où la durée de vie calculée est la plus courte se trouvent dans le métal fondu. Or le métal fondu est, a priori, plus résistant en fatigue que le métal de base. 
Pour identifier les paramètres du modèle à 2 échelles nous avons utilisé des courbes $S-N$ établies en traction. Or la sollicitation de flexion et la concentration de contrainte due à la soudure induisent un gradient de contrainte important dont les effets en fatigue ne sont pas pris en compte ici.

Certains paramètres ne sont pas pris en compte dans la simulation, en particulier les contraintes résiduelles, même faibles, peuvent modifier la durée de vie.

Les géométries que nous avons choisies du côté dessous peuvent paraître sévères. Cependant, les valeurs d'angles et de rayons choisis sont représentatives des mesures, et les durées de vie calculées pour ces géométries sont proches des durées de vie expérimentales.

\section{Conclusion}

La méthode mise en œuvre dans cette étude pour calculer la durée de vie à l'amorçage d'une fissure de fatigue dans un joint soudé de type naval se décompose en deux parties : un calcul de structure non-linéaire par élémentsfinis puis un post-traitement utilisant un modèle d'endommagement à deux échelles.

Le calcul par éléments-finis prend en compte le comportement non-linéaire du matériau, la géométrie locale du cordon de soudure et une variation de la limite d'élasticité dans le métal fondu et la ZAT.

Le post-traitement est utilisé uniquement lorsqu'une adaptation élastique se produit au cours des 100 premiers cycles de chargement. On considère ici que toute la soudure a les mêmes propriétés en fatigue que le métal de base, ce qui est une hypothèse très conservative.

Les données expérimentales nécessaires pour l'application de cette méthode sont limitées. Pour le métal de base, nous avons utilisé les résultats suivants : un essai de traction monotone jusqu'à rupture, un essai de traction compression cyclique et deux courbes $S-N$ pour deux rapports de charge différents. Pour la soudure, nous avons utilisé des mesures de dureté dans les différentes zones et des mesures de profil du cordon.

La comparaison avec les durées de vie expérimentales obtenues en flexion est encourageante. Les durées de vie calculées sont inférieures aux durées de vie expérimentales et l'écart entre les deux est en partie expliqué par les hypothèses conservatives.

Des essais de fatigue sous chargement d'amplitude variable ont également été réalisés, les calculs des durées de vie correspondantes sont en cours.
L'application de cette méthode à un autre procédé de soudage est actuellement à l'étude.

Remerciements. Les auteurs remercient le Conseil Régional de Bretagne pour le financement de ce travail. Ils remercient également DCN pour la fourniture d'échantillons soudés.

\section{Références}

[1] J. Lemaitre, I. Doghri, Damage 90: a post processor for crack initiation, Comput. Methods Appl. Mech. Engrg. 115 (1994) 197-232

[2] A. El Malki Alaoui, Influence du chargement sur la propagation en fatigue de fissures courtes dans un acier de construction navale, Thèse de doctorat, Université de Metz, 2005

[3] H.P. Lieurade, I. Huther, D. Lebaillif, Évaluation de la qualité des soudures vis-à-vis de la résistance à la fatigue, Mécanique \& industries 6 (2005) 133-143

[4] P. Allain, Analyse de contraintes résiduelles sur des témoins soudés en acier E 355 fournis par l'ENSIETA, Rapport d'essais, DCN CESMAN, 2006

[5] P. Paris, F. Erdogan, A critical analysis of crack propagation laws, J. Basic Eng. Trans. ASME 85D (1963) $528-534$

[6] I.S. Raju, J.C. Newman, Stress intensity factors for a wide range of semi-elliptical surface cracks in finite thickness plates, Engng. Fracture Mechs. 11 (1979) 817-829

[7] F. Cortial, A. Poher, C. Le Gall, Dossier matériau au 09/96 de l'acier S355NL, Compte rendu d'étude, DCN CESMAN, 1996

[8] J. Lemaitre, J.-L. Chaboche. Mécanique des matériaux solides, Dunod, Paris, $2^{\mathrm{e}}$ édition, 2004

[9] J. Lemaitre, R. Desmorat, Engineering Damage Mech., Springer Verlag, Berlin, 2005

[10] J.D. Eshelby, The determination of the elastic field of an ellipsoidal inclusion, related problems, Proc. Roy. Soc. A241 (1957) 376-396

[11] R. Desmorat, Modélisation et estimation rapide de la plasticité et de l'endommagment, Mémoire d'habilitation à diriger des recherches, Université Pierre et Marie Curie, 2000

[12] P.-M. Lesne, S. Savalle. An efficient jump technique for viscoplastic structure calculations involving large number of cycles, in 2nd International Conference on Computational Plasticity, Barcelone, 1989 